\title{
Endocrine therapy for the treatment of leptomeningeal carcinomatosis in luminal breast cancer: a comprehensive review
}

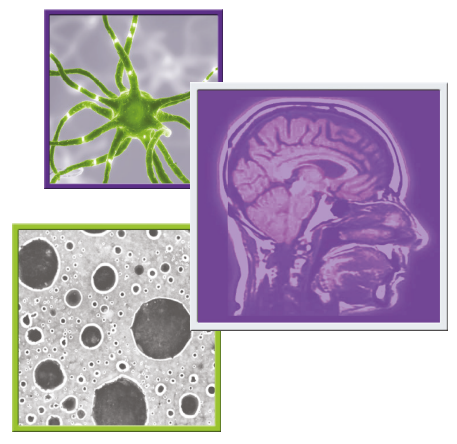

\author{
Leonor Fernandes*,1(i), Leonor Vasconcelos de Matos'(iD), Débora Cardoso', Marlene \\ Saraiva $^{2}$ (D), Renata Medeiros-Mirra ${ }^{3}$ (D) Andreia Coelho', Helena Miranda ${ }^{1}$ (iD) \& Ana \\ Martins ${ }^{1}$ \\ ${ }^{1}$ Department of Medical Oncology, Hospital São Francisco Xavier, Centro Hospitalar Lisboa Ocidental, 1449-005 Lisboa, Portugal \\ 2Department of Neurology, Hospital Egas Moniz, Centro Hospitalar Lisboa Ocidental, 1349-019 Lisboa, Portugal \\ ${ }^{3}$ Cardiff School of Dentistry, College of Biomedical \& Life Sciences, Cardiff University, Cardiff CF10 3AT, Wales, UK \\ *Author for correspondence: Tel.: +351964 553 711; fernandes.leonor@gmail.com
}

Leptomeningeal disease (LMD) represents a devastating complication of advanced breast cancer ( $A B C$ ), with survival of $<5$ months with multimodal treatment. The role of endocrine therapy (ET), due to its favorable toxicity profile and first-line indication in luminal $A B C$, appears promising in the setting of $L M D$, where symptom stabilization and quality-of-life preservation are the main goals; however, evidencedbased data are lacking. We conducted a thorough review of published evidence, aiming to investigate the role of ET in LMD treatment in luminal ABC. Twenty-one of 342 articles, evaluating 1302 patients, met inclusion criteria. ET use was rarely reported. New targeted agents show CNS activity. Research is lacking on the role of ET and targeted agents in BC-LMD treatment.

First draft submitted: 6 September 2020; Accepted for publication: 2 October 2020; Published online: 20 October 2020

Keywords: breast cancer $\bullet$ endocrine therapy $\bullet$ hormonal therapy $\bullet$ leptomeningeal disease $\bullet$ luminal-like breast cancer • meningeal carcinomatosis • systemic therapy

Leptomeningeal disease (LMD) is characterized by tumor cells spread within the leptomeninges or the subarachnoid space [1]. Diagnosis can be challenging because signs and symptoms may be subtle. The presence of malignant cells in the cerebral spinal fluid (CSF) (specificity $>95 \%$, sensibility 45-75\% [2]) and/or magnetic resonance imaging (MRI) with gadolinium (the accuracy of which is still debatable) consistent with CSF dissemination and clinical suspicion confirms the diagnosis. LMD is mainly a late-stage complication but can be an inaugural diagnosis $(10 \%)[3,4]$.

In breast cancer (BC), LMD has an estimated incidence of 5\% [5,6]. Triple negative (TN) subtype, lobular histology and ventricle disruption during surgical excision of brain metastasis are associated with a higher risk for LMD-BC $[1,5,7]$. Distribution by subtype is almost equivalent in proportions: $17-37 \%$ for TN BC, $19-40 \%$ for HER2 positive (HER2+) BC and 35-50\% for luminal BC [hormone receptor positive (HR+) and HER2 negative (HER2-)] [8-13].

Patients with LMD have a dismal prognosis. At diagnosis, $80 \%$ are symptomatic with severely impaired functional status and quality of life $[5,6,14]$. Median survival without treatment is $6-8$ weeks; with multimodal treatment, it usually does not exceed 5 months $[1,5,8,12,14]$. At 1 year, less than $25 \%$ are alive $[1,8]$.

No prognostic score has been validated to help clinicians with stratifying patients or providing the appropriate therapeutic approach $[7,8,12]$. The most consistent favorable prognostic factor seems to be a good performance status (PS) at diagnosis $[5,8,11,15-18]$.

Given the bleak prognosis of LMD, stabilizing neurologic symptoms, improving quality of life and preventing or delaying further disability are the goals of treatment [1]. A multimodal approach has been suggested to be the key for a sustained response [12,19,20]. Accordingly to the European Society for Medical Oncology recommendations, surgery, radiotherapy (RT), systemic anticancer therapy (SACT), intrathecal therapy (ITT) and supportive therapy 
should be used in conjunction for patients with good performance status [1]. Surgery is mostly indicated in cases that require relief of intracranial pressure, placement of a ventriculoperitoneal shunt or insertion of an Ommaya reservoir [21,22].

RT has an important role in highly symptomatic and bulky disease, and it seems it can improve results in the setting of a multimodal approach. However, its effect on survival and quality of life have not been well established [13,19,23-25]. Furthermore, it seems that the molecular landscape and tumor microenvironment of CNS metastasis may have implications in terms of RT response, which in the future could dictate multimodality approaches [26-28].

Most retrospective LMD studies suggest that SACT improves survival $[1,5,11,12,29]$. However, significant challenges to its use have been pointed out, including limited tested regimens in this setting, the assumption that most regimens have limited efficacy in CNS metastasis and the paucity of additional available treatment lines. The blood-brain barrier (BBB) is cited as the principal factor for the limited CNS activity of most therapeutic regimens [26], but several authors suggest that in the presence of brain metastasis, the integrity of the BBB has been compromised, which could explain the survival benefit provided by SACT [30,31]. Furthermore, LMD itself has been shown to increase BBB permeability [7], and these patients are often subjected to whole-brain RT, which disrupts BBB organization and consequently increases concentration of systemic agents in CSF [7,31].

Administration of agents directly in the CSF bypasses BBB and, in theory, ensures therapeutic levels of antineoplastic agents, although diffuse distribution of these implies absence of an obstruction to CSF flow [24]. These are the principles of ITT that have been the mainstay for BC-LMD treatment in most European centers [17], yet its value is not well established [32]. Furthermore, its use is not recommended in the presence of hydrocephalus (because, due to impaired CSF flow, it augments the risk of neurotoxicity, furthering intracranial pressure and consequently brain herniation and ischemia) $[4,22,33]$ or nodular disease only (due to limited diffusion of ITT agents into the subependymal tissue) $[1,7,24,34]$. ITT also involves invasive procedures and has possible serious adverse effects [24,29,35], even if it seems not to negatively affect quality of life [25,32].

Endocrine therapy (ET) alone or in combination with other agents is the standard first-line treatment for advanced luminal BC (luminal ABC) unless patients are endocrine resistant or present with visceral crisis [36]. ET is extremely effective in controlling bone disease [37,38], including disease of the skull, because bone is the most common place for luminal BC metastasis [39]. Regarding CNS metastasis, patients have been excluded from most trials. Furthermore, most studies on LMD have not examined BC exclusively nor have they been dedicated to researching the value of ET, with many focusing mainly on ITT [30,32,40], chemotherapy regimens [41-43] and RT $[19,23,44]$.

Some studies suggest that there is a role for ET in luminal ABC with CNS metastasis, including LMD [15,31,45-47]. There is evidence that hormonal agents can cross the BBB: tamoxifen has a good CSF bioavailability and can also modulate p-glycoprotein, which are important for achieving CNS activity [48,49]; likewise, letrozole penetrates the BBB better than anastrozole [50] and seems to have potential tumor selectivity by achieving greater levels in tumor areas [51]. It can be inferred that demonstration of efficacy in controlling CNS disease comprises control of LMD, and thus results from CNS disease should guide treatment approaches to this rare manifestation of advanced $\mathrm{BC}$ $(\mathrm{ABC})$.

Decisions about LMD treatment lack evidence-based practice. The rarity of the disease along with the usually poor clinical performance due to symptom burden brought by LMD limits randomized trial conduction and prospective data gathering. The aim of this review was to investigate the role of ET in the treatment of LMD in patients with luminal BC.

\section{Methods}

\section{Study question \& inclusion criteria}

This study was designed to evaluate published evidence underlying ET options for LMD in luminal ABC. The research question was set using the Population-Intervention-Comparator-Outcome-Study Design framework [52].

The population of interest included individuals aged $>18$ years with diagnosis of LMD in the context of luminal $\mathrm{BC}$ for whom ET was given after diagnosis. All articles featuring this population, even if submitted to other treatments or without available outcomes and survival for this group, were eligible for inclusion.

Studies featuring other tumors were not excluded if the information required was presented. Studies using ET in combination with targeted agents for HER2 disease were excluded due to the possible confounding effect. 
Figure 1. Search strategy according to Preferred Reporting Items for Systematic Reviews and Meta-Analysis method.

BC: Breast cancer; ET: Endocrine therapy; LMD: Leptomeningeal disease; TN: Triple negative.

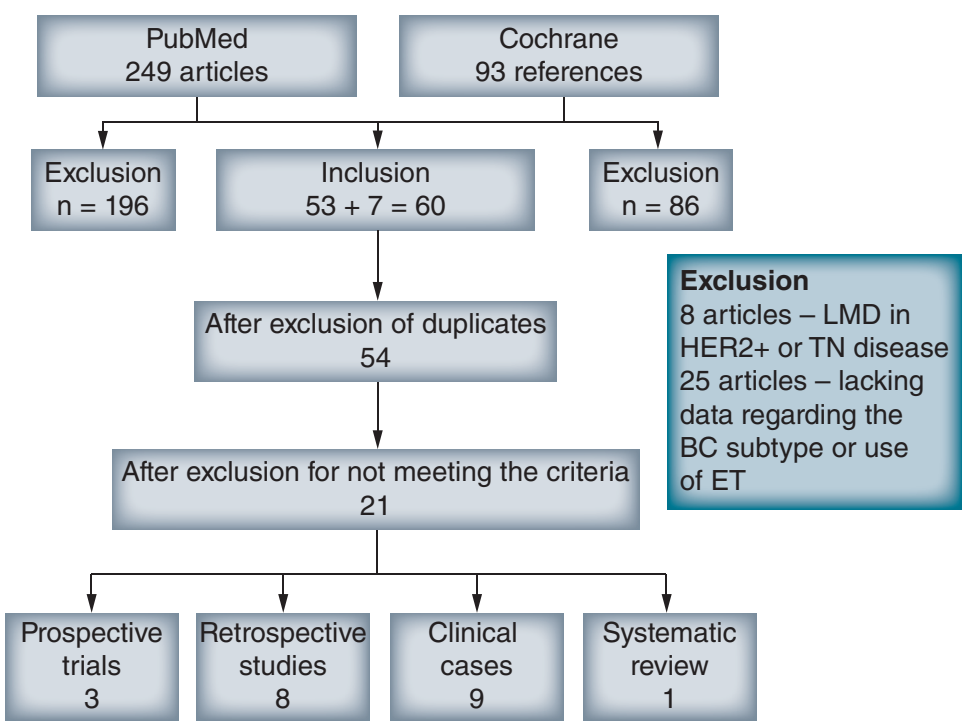

\section{Literature review}

This review was performed independently in accordance with the Preferred Reporting Items for Systematic Reviews and Meta-Analysis (PRISMA) guidelines [53]. Eligible articles published between 2000 and as late as July 2020 were retrieved by searching PubMed and COCHRANE. The search strategy was restricted to publications in English and Portuguese and included all publications with BC and LMD (or carcinomatosis or metastasis) and ET (or hormonal therapy or tamoxifen or letrozole or anastrozole or fulvestrant or exemestane). All study types were permitted, including randomized and nonrandomized interventional studies, observational studies (controlled or uncontrolled), systematic reviews and clinical cases or case series.

\section{Data collection \& statistical analysis}

For each study, the following data were collected: year of publication; first author; patient age at diagnosis; Karnofsky Performance Status (KPS)/Eastern Cooperative Oncology Group (ECOG)-PS; estrogen, progesterone and HER2 status; type of ET used (when available); and other therapeutic strategies used before and after LMD diagnosis. Whenever possible, we also gathered the following data: time from breast cancer diagnosis until LMD diagnosis (TLMD), progression-free survival corresponding to the time each ET was used before changing line due to progression, quality of life, treatment toxicity and overall survival (OS) for LMD. OS was calculated as time from LMD diagnosis until death or last contact (OS_LMD). We performed a simple one-sample comparative analysis, with the nonparametric Wilcoxon test, using R statistical software version 4.0.2 [54].

\section{Results}

Our search retrieved a total of 342 articles of which 21 studies were included (selection is detailed in Figure 1). These evaluated 1302 patients, of which 161 of 473 with luminal ABC-LMD received ET after diagnosis. We organized data in four groups: prospective studies (Table 1), retrospective studies (Table 1), clinical cases/case series (Table 2) and systematic reviews (Table 1). Because of the substantial heterogeneity of data, we only compared median OS of the group of clinical cases against reference median OS for LMD (described in more detail subsequently).

\section{Prospective trials}

Three trials were included in this group (Table 1). In a randomized study from 2004 with 35 patients, Boogerd et al. compared ITT with methotrexate combined with SACT versus SACT alone. Fourteen patients received ET, seven combined with ITT (experimental group) [29]. Agents used were tamoxifen, orimeten, megestrol and fluoxymesterone. This trial showed better OS for patients in the control group (SACT only), but OS was not detailed according to the type of therapy used (OS: 18.3 weeks vs 30.3 weeks, $95 \%$ CI: 5.5-34.3 weeks).

The only phase III trial in BC-LMD, DEPOSEIN, was recently published and enrolled 73 patients (37 in the control group and 36 in the experimental group) with the aim of comparing ITT with SACT versus SACT alone [32]. Although 39 patients had luminal ABC, only two were treated with ET. After LMD progression, 19 more 
in the control group and 10 in the experimental group received further systemic agents, but no additional detail is given [32]. The experimental group almost doubled OS (OS: 4.0 months [95\% CI: 2.2-6.3] vs 7.3 months [95\% CI: 3.9-9.6], hazard ratio [HR]: $0.85,95 \%$ CI: 0.53-1.36), suggesting that ITT with SACT is the best approach for LMD. However, this was a small, open-label trial without stratifications, leading to unbalanced groups [32], and the pattern of LMD disease (nodular vs linear) was not accounted [32].

Recently, the first prospective trial to evaluate the efficacy and safety of immune checkpoint inhibitors in patients with LMD of any solid tumor was published [55]. Brastianos et al. tested pembrolizumab in 20 patients, 17 with BC. Of those with BC, seven were HR+/HER2-, and two received pembrolizumab concurrently with ET (fulvestrant and letrozole). The primary endpoint of this study was met, and at 3 months, 12 of 20 patients were alive including four patients from the group of luminal BC-LMD patients (OS 3.4-14.6 months). Outcomes of the two patients with concurrent pembrolizumab and ET are not disclosed [55].

\section{Retrospective studies}

In this group, eight studies met the inclusion criteria, although most did not detail the variables under study (Table 1). Of 403 luminal ABC patients, 135 received ET after LMD diagnosis.

In 2013, three retrospective studies on this subject were published. LeRhun et al. studied a cohort of 103 patients diagnosed between 2007 and 2011 and treated with intrathecal cytarabine combined with other treatments [15]. 44 had luminal ABC-LMD, and 15 started concomitant ET. The authors reported a median OS of 3.8 months (1 day2.8 years) for the whole cohort, but in univariate and multivariate analysis, both non-TN tumors ( $\mathrm{p}=0.0139)$ and initial treatment that included ET $(\mathrm{P}=0.0238)$ were significantly associated with a better OS.

Comte et al. also published a retrospective study with 66 patients diagnosed between 2000 and 2012, of which 45 had HR+ BC-LMD, although it is not specified if they are all luminal [14]. Their aim was to evaluate benefits of ITT with thiotepa. SACT was used in combination with intrathecal thiotepa, $90 \%$ patients had been previously treated with SACT regimens and ET was used only in nine patients (14\%). Median OS was 4.5 months (0.1-50 months) for the cohort, and 12 patients (18\%) had OS $>1$ year. Univariate analysis showed that high grade tumors, $>3$ prior lines of chemotherapy and PS $>2$ were poor prognostic factors [14]. ET agents were not specified, but use of hormonal treatment $(n=9 / 66)$ was not associated with better OS (univariate analysis, $p=0.15$ ).

Torrejón $e t$ al. also studied implications of BC subtypes for the development of LMD [10]. The retrospective cohort of 38 BC patients included 19 luminal ABC (seven luminal A and 12 luminal B). Only one was treated with an ET (anastrozole) in addition to other SACT. The authors concluded that the use of SACT was related to a better prognosis and that luminal $\mathrm{ABC}$ patients had longer TLMD but worse nonsignificant OS, compared with TN (OS: luminal B 1.3 months, luminal A 2.7 months, HER2+ 3.0 months and TN 3.1 months, $\mathrm{p}=0.296$ ).

Abouharb et al. published a study that included 233 patients with BC-LMD diagnosed between 1997 and 2012 [11]. The aim was to characterize clinical features and outcomes of patients with LMD based on BC subtypes. Survival for luminal BC patients was better than for TN BC but worse than for HER2+ BC (OS 3.7 vs 2.2 months and 4.4 months, respectively). Forty-four of 67 luminal BC patients received SACT and 19 (28\%) received ET, but specific survival for the latter group is not detailed. Multimodal treatment was used in most patients, and only $10 \%$ proceeded to supportive care immediately after LMD diagnosis. Patients who received SACT (in all molecular subtypes) had significantly better OS (for all: OS 6.4 vs 1.7 months; HR: 0.31 ; 95\% CI: $0.24-0.42 \mathrm{p}<0.001$; luminal group: HR: $0.41 ; 95 \%$ CI: $0.24-0.69, \mathrm{p}=0.001$ ).

In 2017, Kingston et al. studied 182 patients diagnosed between 2004 and 2014, of whom 90 (49.5\%) had luminal ABC with LMD [56]. The authors aimed to evaluate potential predictors of survival. Seven patients received ET, four patients concomitantly with RT (2) and ITT (2); three patients received supportive care but maintained hormonal treatment. OS was 5.4 months, and progression-free survival was 3.9 months for all treatment approaches. The group that received SACT as the first strategy had the best OS ( 8.8 months), but the authors only mentioned chemotherapy and did not detail all regimens used. Their conclusion was that there is no current indication to deviate from standard ABC regimens when treating LMD because patients who received ITT or palliative care alone had the worst outcomes.

In the same year, Niwinska et al. studied retrospectively the factors affecting survival of BC-LMD in a cohort of 187 patients diagnosed between 1999 and 2015 [12]. 32 patients of 75 with luminal ABC-LMD received ET during the course of their disease, but the authors did not detail which agent was used or the OS for this group. They concluded that older age, luminal ABC (HR: 0.64; 95\% CI: 0.461-0.887, $\mathrm{p}=0.007$ ) and good PS were linked to better prognosis, but the use of multimodal treatment, specifically SACT and RT, was the strongest positive factor. 
Patients with the best prognosis $(n=24)$ achieved a median OS of 9.6 months (95\% CI: 4.3-14.9). The authors did not detail median OS for luminal patients.

The retrospective study by Griguolo et al. aimed to show the prognostic value of systemic therapy and comprised 153 patients diagnosed between 2002 and 2017 [18]. A total of 110 patients received SACT, 78 patients had luminal ABC, but only 44 received ET. Median OS for the luminal ABC group was 3.2 months, but specific outcomes for the ET group were not explored [18]. This study also concluded that patients with deteriorated PS were less likely to receive treatment, in particular SACT and RT. Also, in the group of HER2- patients ( $\mathrm{n}=101$, HR+/HER2and TN), use of both SACT (HR: 0.16; 95\% CI: 0.1-0.27; $<$.001) and ITT (HR: 0.42; 95\% CI: 0.27-0.65; $\mathrm{p}<0.001$ ) were significantly related with better prognosis.

The only retrospective study that specifically addresses ET in BC-LMD was published in 2019; the primary aim of Bergen et al. was to evaluate the impact of ET on the survival prognosis of patients with luminal-ABC and brain metastasis [45]. Their cohort had 198 patients with brain metastasis diagnosed between 1990 and 2017, of whom 30 concomitantly had LMD. Only eight of these received ET; OS was double for these patients compared with those who did not receive ET along the treatment ( 7 months vs 3 months, $\mathrm{p}=0.012$ ). Overall, there were no significant differences between agents used (aromatase inhibitors, tamoxifen or fulvestrant). The authors concluded that ET significantly improves survival in BC-CNS metastasis and might particularly be considered in the presence of nodular LMD.

\section{Clinical cases}

We found nine clinical cases reporting 12 women with BC-LMD treated ET (Table 2) [46,47,58-64]. Nine patients received multimodality treatment after LMD diagnosis in addition to ET, RT (7), ITT (4) and chemotherapy (5). Eight patients received multiple endocrine agents, and of these, two were also treated with CDK 4/6 inhibitors. All had good clinical responses, and the median OS was 25.5 months (12-120 months; two cases had no OS detailed). Median TLMD was 35.5 months (min-max: 0-216 months), and in four cases LMD was the inaugural diagnosis of BC.

\section{Survival analysis}

Analysis of OS_LMD was done only for the group of clinical cases; therefore, it is based on only 12 patients with luminal ABC-LMD, treated with ET combined or alone and compared with three reference OS_LMD. For comparison, we choose three OS that we thought could be representative of the median: OS for BC-LMD (median OS from the retrospective studies reviewed here [ 4.3 months]), the best median OS found in this review (median OS achieved by the best prognostic group in the Niwinska et al. cohort [9.6 months]) and the median OS of the experimental group in the DEPOSEIN trial (7.3 months).

The median OS_LMD from the clinical cases group was significantly different from all three comparisons (25.5 vs 9.6 months, 4.3 and 7.3 months, respectively, with adjusted $\mathrm{p}<0.01$ for all of them) (Table 3).

\section{Systematic Reviews}

Only one publication from 2016 was included in this group (Table 1). Lee et al. conducted a systematic review and pooled analysis, of seven case series and 25 case reports, to compare outcomes of ET, chemotherapy and ITT in BC-LMD [57]. There were only seven patients in the ET group, which comprised only patients from clinical cases. The Kaplan-Meier method showed no differences in survival. The ET group had the longest median OS (65 vs 52 weeks in the systemic chemotherapy vs 41 weeks in the ITT group). One patient who received ET exhibited the longest survival of approximately 8.5 years. Five of the clinical cases from this work are the same in our group of clinical cases.

\section{Discussion}

Results from the studies reviewed

The first European guidelines for LMD were published in 2017 and highlighted the scarcity of evidence in this area [1]. Also, in the setting of $\mathrm{ABC}$, metastatic CNS disease has historically been excluded from most clinical trials. This reality is changing, and in recent years trials have been designed to specifically approach CNS metastasis [55,65], some of them, in particular those in HER2+ disease [66,67], with promising results. Despite this, LMD continues to be understudied, and evidence for optimal treatment in BC-LMD is still limited [67]. Use of SACT has been consistently linked to better prognosis [12,14,56], but in Europe, ITT is the mainstay in LMD [17]. 







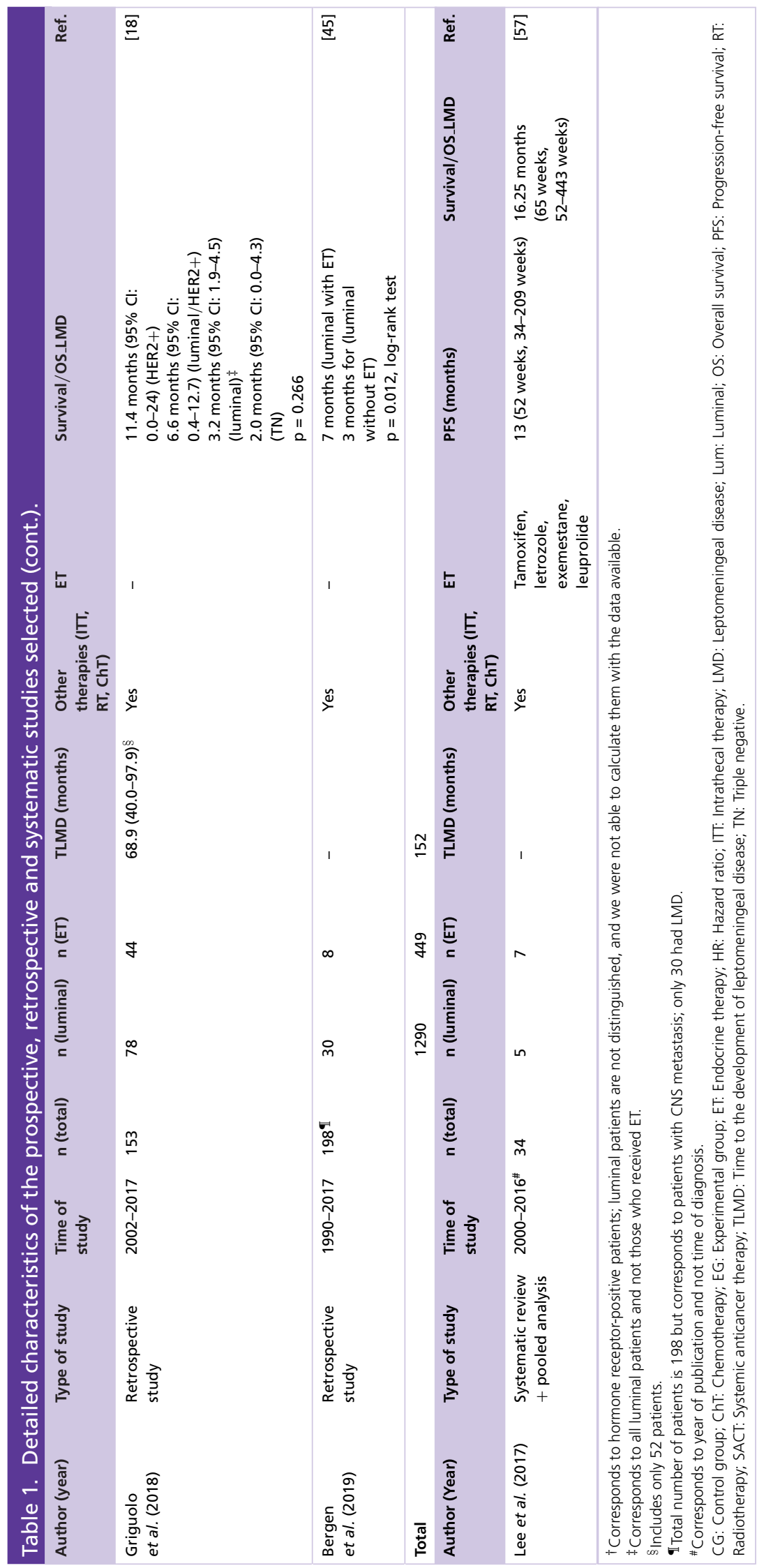




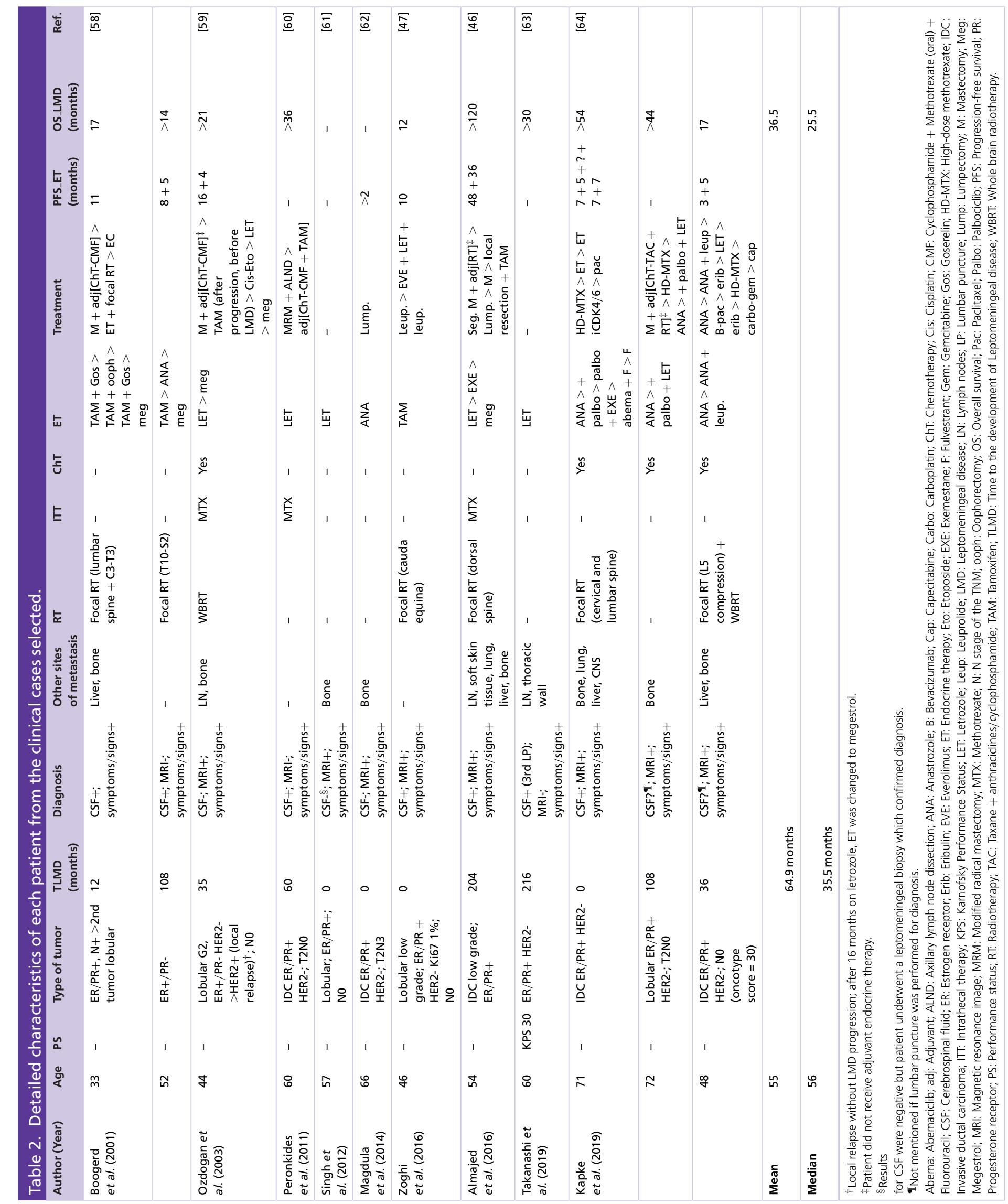


Table 3. Median overall survival comparative analysis with the Wilcoxon test.

\begin{tabular}{|lll|}
\hline Cohorts & Median overall survival (months) & Wilcoxon test (p) \\
\hline Le Rhun et al. [15] & 3.8 & \\
\hline Comte et al. [14] & 4.5 & \\
\hline Abouharb et al. [11] & 4.4 \\
\hline Griguolo et al. [18] & 3.9 \\
\hline Kingston et al. [56] & 5.4 & $\mathrm{p}<0.01$ \\
\hline Niwinska et al. [12] & 4.3 & \\
\hline Median & $\mathbf{4 . 3}$ & \\
\hline Experimental group DEPOSEIN trial & $\mathbf{7 . 3}$ & \\
\hline Best prognostic group (Niwinska et al.) & $\mathbf{9 . 6}$ & \\
\hline Group of clinical cases & $\mathbf{2 5 . 5}$ & \\
\hline
\end{tabular}

The most important works published in this setting are the recent DEPOSEIN phase III trial [32] and the phase II trial of pembrolizumab in LMD [55]. DEPOSEIN, in our opinion, reinforces the idea that BC-LMD requires a combined modality approach and that ITT should be used in subsets of patients, which is in accordance with expert recommendations [24]. In this trial, although approximately $50 \%$ of these patients had luminal ABC, only a small minority received ET. However, evidence shows that if BC-LMD is treated according to its molecular characteristics, better results are achieved [3,40,55,65,68].

CNS metastasis occur less frequently and usually later in the course of HR+/HER2- disease compared with other BC subtypes [69,70]. Thus, evidence on the use of ET in luminal ABC-LMD is lacking. In all retrospective studies analyzed, the trend is the same, whenever SACT is used, ET is not the first choice and we cannot assure that this happens due to endocrine resistance or lack of available lines. We speculate that clinicians consider that ET is not a suitable option for CNS metastasis [67] and opt for ITT or chemotherapy, even if CNS metastasis remains $\mathrm{HR}+$ in more than two thirds of patients [71-73]. Furthermore, underlined studies were performed in very uneven time periods and thus a great heterogeneity in treatment approaches can be observed, reflecting the great changes that $\mathrm{ABC}$ treatment has undergone in the last decades yet missing the advent of the CDK 4/6 inhibitors era.

In this review we have gathered data showing that subsets of patients have almost double the median OS in BC-LMD [12,45] and that use of ET in LMD can achieve prolonged survival rates compared with the usual reported outcomes [46,47,58-64,74]. Our statistical comparison showed significant differences between OS_LMD, suggesting that risk stratification could help identifying the most suitable patients for ET.

Only a few prospective ongoing studies are dedicated to the use of ET in luminal ABC-LMD, and most of them are in BC patients with CNS metastasis, and LMD patients with stable disease were not excluded [7,66]. Less than a handful are with newer ET agents (Z-endoxifen or elacestrant), and the majority are with ET combined with other agents such as the CDK4/6 inhibitors abemaciclib, palbociclib and ribociclib; PI3K inhibitors; the mTOR inhibitor everolimus; VEGFR inhibitors cabozantinib and lenvantinib; and IGF receptor antibodies (xentuzumab and BMS-754807) [7,66].

\section{Endocrine therapy combined with other agents}

Because LMD is a late event in luminal BC, it is possible that, due to endocrine resistance, ET cannot be used alone in this setting. With the introduction of CDK4/6 and mTOR/AKT/PI3K inhibitors, more therapeutic options for luminal $\mathrm{ABC}$ are available, which can somewhat overcome this problem. Palbociclib, abemaciclib, everolimus and buparlisib are all able to cross the $\mathrm{BBB}[7,26,65,66,75]$, and alpelisib appears to be active against parenchymal brain metastasis [76].

An exploratory arm in a recent nonrandomized multicohort phase II trial (NCT02308020) confirmed that abemaciclib and its metabolites achieve therapeutic concentrations in CSF approximating that in plasma and can control BC-LMD, achieving longer OS than that seen in historical controls [65]. Although, data suggest that these were heavily pretreated patients (five received a median of four SACT lines, all including chemotherapy and some ET and target agents), which is one of the problems involved in the management of LMD. Nonetheless, in the cohort of luminal BC patients with parenchymal brain metastasis, concomitant abemaciclib and ET achieved better responses in terms of intracranial clinical benefit (35.7 vs $21.1 \%$ ), suggesting a synergistic action of these drugs in CNS disease [65]. 
Other targeted agents, such as AKT inhibitors, are promising for treatment of BC patients with CNS metastasis because some of these molecules are able to cross the $\mathrm{BBB}$ [77] and seem to prolong ET response in endocrine-resistant patients (demonstrated by the FAKTION trial of capivasertib with fulvestrant) [78].

Also, regarding mTOR/AKT/PI3K inhibitors, it seems that PI3K-mutant patients have a higher probability of developing CNS metastasis [79,80], which is something that requires further exploration in BC-LMD [76]. Specifically, as identified by Le Rhun et al., single nucleotide polymorphisms in the PI3KR1 gene seem to be associated with CNS metastasis [79]. Several ongoing trials are exploring efficacy of PI3K inhibitors. The latest results published from the ongoing BYLieve trial (NCT03056755) showed that alpelisib after CDK4/6 inhibition shows clinically meaningful efficacy and manageable toxicity [81]. However, alpelisib has only been approved for PIK3CA-mutated patients, in contrast to capivasertib and everolimus, which have demonstrated clinical benefit independent of PI3K mutations [78].

Regarding the best sequence to maximize results, data suggest that abemaciclib combined with ET followed by mTOR/AKT/PI3K inhibitors with ET could be the best option for HR+/HER2- ABC patients with CNS metastasis, but further and more mature data are needed to confirm this assumption.

Adding complexity to SACT options for these patients is the not rare event of discordance in hormone receptor status between primary tumor and CNS metastasis, with an HR+ primary switching to negative in one-fifth to one-third of cases $[72,73,82]$. New diagnostic techniques, such as analysis of circulating epithelial tumor cell DNA in CSF, could help increase diagnostic sensitivity and specificity and establish $\mathrm{ABC}$ subgroups as well as driver and resistance mutations, which could help dictate therapeutic choices [83].

Immunotherapy is also another emerging field that might bring hope to patients with LMD. It seems that CNS metastasis responds to these agents independent of PDL1 status, and even if immunotherapy seems more suitable for the TN BC subtype, targeted agents such as CDK4/6 and mTOR/AKT/PI3K inhibitors seem to alter immunogenicity in $\mathrm{HR}+$ patients, thus opening further possibilities, such as combining trials of target agents with immunotherapy or sequential therapies of these agents $[84,85]$.

We hope that in a near future, more targeted trials in LMD are designed that can clarify the best treatment option for subtypes of BC-LMD (Supplementary Table 1 lists ongoing trials in LMD).

\section{Conclusion \& future perspective}

This review has highlighted the urgent need for clinical trials in BC-LMD that have been stratified on tumor biology, which is already being done for HER2+ tumors, with promising results. Overall, we can say that there is still a great lack of knowledge in this area.

There is a need to identify in which patients a multimodal therapeutic approach should be considered. We postulate that ET, with or without other agents, with its ease of use, advantageous side effect profile and highly probable improvement in quality of life, should be considered as a first choice for the treatment of luminal ABCLMD, especially in inaugural LMD. The future will see a multimodality approach tailored for BC subtypes and possibly different LMD characteristics.

Precision medicine approaches in BC-LMD may improve outcomes. One size does not fit all, and it is urgent that a more personalized approach to BC-LMD treatment is developed to improve the poor prognosis.

\section{Executive summary}

- Leptomeningeal disease (LMD) is rare but a devastating complication of breast cancer (BC), and its optimal therapy is still not defined.

- Diagnosis is made by cerebral spine fluid cytology or magnetic resonance imaging with multifocal signs/symptoms suggestive of LMD.

- Prognosis without treatment is 6-8 weeks; multimodality treatment (surgery, systemic anticancer treatment [SACT], intrathecal treatment, radiotherapy) can increase prognosis to 5 months.

- Use of SACT is associated with better outcomes.

- In the setting of luminal BC, endocrine therapy, with or without CDK4/6 inhibitors, may play a role in LMD because it is the standard-of-care treatment for advanced BC. It is a well-tolerated therapy with a favorable toxicity profile. However, evidence is scarce for its use in the setting of BC-LMD. 


\section{Supplementary data}

To view the supplementary data that accompany this paper please visit the journal website at: www.futuremedicine.com/doi/sup $\mathrm{pl} / 10.2217 / \mathrm{cns}-2020-0023$

\section{Author contributions}

LF: concept and literature review, writing of the original manuscript. LM: concept and literature review; writing: review and editing. DC: supervision; writing: review and editing. MS: supervision; writing: review and editing. RM-M: statistical analysis; writing: review and editing. AC: supervision; writing: review and editing. HM: supervision; writing: review. AM: supervision.

\section{Financial \& competing interests disclosure}

The authors have no relevant affiliations or financial involvement with any organization or entity with a financial interest in or financial conflict with the subject matter or materials discussed in the manuscript. This includes employment, consultancies, honoraria, stock ownership or options, expert testimony, grants or patents received or pending, or royalties.

No writing assistance was utilized in the production of this manuscript.

\section{Acknowledgments}

The authors acknowledge Dr Francisca Costa for her help in reviewing language and submitting this article.

\section{Open access}

This work is licensed under the Attribution-NonCommercial-NoDerivatives 4.0 Unported License. To view a copy of this license, visit http://creativecommons.org/licenses/by-nc-nd/4.0/

\section{References}

Papers of special note have been highlighted as: $\bullet$ of interest; $\bullet \bullet$ of considerable interest

1. Le Rhun E, Weller M, Brandsma D et al. EANO-ESMO clinical practice guidelines for diagnosis, treatment and follow-up of patients with leptomeningeal metastasis from solid tumours. Ann. Oncol. 28(Suppl. 4), iv84-iv99 (2017).

2. Angus L, Martens JWM, Van Den Bent MJ et al. Novel methods to diagnose leptomeningeal metastases in breast cancer. Neuro Oncol. 21(4), 428-439 (2019).

3. Brastianos P, Davies MA, Margolin K, Yu HA. Modern management of central nervous system metastases in the era of targeted therapy and immune oncology. Am. Soc. Clin. Oncol. Educ. Book 39, e59-e69 (2019).

4. Wang N, Bertalan MS, Brastianos PK. Leptomeningeal metastasis from systemic cancer: Review and update on management. Cancer 124(1), 21-35 (2018).

5. Franzoi MA, Hortobagyi GN. Leptomeningeal carcinomatosis in patients with breast cancer. Crit. Rev. Oncol. Hematol. 135, 85-94 (2019).

- A comprehensive review about leptomeningeal disease in breast cancer.

6. Scott BJ, Oberheim-Bush NA, Kesari S. Leptomeningeal metastasis in breast cancer-a systematic review. Oncotarget 7(4), 3740-3747 (2016).

7. Figura NB, Rizk VT, Armaghani AJ et al. Breast leptomeningeal disease: a review of current practices and updates on management. Breast Cancer Res. Treat. 177(2), 277-294 (2019).

-• A comprehensive review of the available published literature on the current state of breast cancer leptomeningeal disease.

8. Gauthier H, Guilhaume MN, Bidard FC et al. Survival of breast cancer patients with meningeal carcinomatosis. Ann. Oncol. 21(11), 2183-2187 (2010).

9. Lee $\mathrm{S}$, Ahn HK, Park YH et al. Leptomeningeal metastases from breast cancer: intrinsic subtypes may affect unique clinical manifestations. Breast Cancer Res. Treat. 129(3), 809-817 (2011).

10. Torrejón D, Oliveira M, Cortes $\mathrm{J}$ et al. Implication of breast cancer phenotype for patients with leptomeningeal carcinomatosis. Breast 22(1), 19-23 (2013).

11. Abouharb S, Ensor J, Loghin ME et al. Leptomeningeal disease and breast cancer: the importance of tumor subtype. Breast Cancer Res. Treat. 146(3), 477-486 (2014).

12. Niwińska A, Pogoda K, Michalski W et al. Determinants of prolonged survival for breast cancer patient groups with leptomeningeal metastasis (LM). J. Neurooncol. 138(1), 191-198 (2018).

13. Znidaric T, Gugic J, Marinko $\mathrm{T}$ et al. Breast cancer patients with brain metastases or leptomeningeal disease: 10-year results of a national cohort with validation of prognostic indexes. Breast J. 25(6), 1117-1125 (2019).

14. Comte A, Jdid W, Guilhaume MN et al. Survival of breast cancer patients with meningeal carcinomatosis treated by intrathecal thiotepa. J. Neurooncol. 115(3), 445-452 (2013). 
15. Le Rhun E, Taillibert S, Zairi F et al. A retrospective case series of 103 consecutive patients with leptomeningeal metastasis and breast cancer. J. Neurooncol. 113(1), 83-92 (2013).

16. Yust-Katz S, Garciarena P, Liu D et al. Breast cancer and leptomeningeal disease (LMD): hormone receptor status influences time to development of LMD and survival from LMD diagnosis. J. Neurooncol. 114(2), 229-235 (2013).

17. Le Rhun E, Rudà R, Devos $\mathrm{P}$ et al. Diagnosis and treatment patterns for patients with leptomeningeal metastasis from solid tumors across Europe. J. Neurooncol. 133(2), 419-427 (2017).

18. Griguolo G, Pouderoux S, Dieci MV et al. Clinicopathological and treatment-associated prognostic factors in patients with breast cancer leptomeningeal metastases in relation to tumor biology. Oncologist 23(11), 1289-1299 (2018).

19. Buszek SM, Chung C. Radiotherapy in leptomeningeal disease: a systematic review of randomized and non-randomized trials. Front. Oncol. 9, 1224 (2019).

20. Rudnicka H, Niwińska A, Murawska M. Breast cancer leptomeningeal metastasis - the role of multimodality treatment. J. Neurooncol. 84(1), 57-62 (2007).

21. Volkov AA, Filis AK, Vrionis FD. Surgical treatment for leptomeningeal disease. Cancer Control 24(1), 47-53 (2017).

22. Lin AL, Avila EK. Neurologic emergencies in the patients with cancer. J. Intensive Care Med. 32(2), 99-115 (2017).

23. Shafie RAE, Böhm K, Weber D et al. Palliative radiotherapy for leptomeningeal carcinomatosis—analysis of outcome, prognostic factors, and symptom response. Front. Oncol. 8, 641 (2019).

24. Le Rhun E, Preusser M, Van Den Bent M et al. How we treat patients with leptomeningeal metastases. ESMO Open 4(Suppl. 2), e000507 (2019).

- A practical guideline for approaching leptomeningeal disease.

25. Dudani S, Mazzarello S, Hilton J et al. Optimal management of leptomeningeal carcinomatosis in breast cancer patients—a systematic review. Clin. Breast Cancer 16(6), 456-470 (2016).

-• A systematic review of current treatment strategies for leptomeningeal disease in breast cancer.

26. O'Sullivan CC, Davarpanah NN, Abraham J, Bates SE. Current challenges in the management of breast cancer brain metastases. Semin. Oncol. 44(2), 85-100 (2017).

27. Venur VA, Chukwueke UN, Lee EQ. Advances in management of brain and leptomeningeal metastases. Curr. Neurol. Neurosci. Rep. 20(7), 26 (2020).

28. Yang TJ, Zhang Z, Young R et al. Abstract CT252: a dose escalation phase I study of concurrent GDC-0084 with radiation therapy for patients with solid tumor brain metastases or leptomeningeal metastases harboring PI3K pathway mutations. Cancer Res. 80(16), CT252-CT252 (2020).

29. Boogerd W, Van Den Bent MJ, Koehler PJ et al. The relevance of intraventricular chemotherapy for leptomeningeal metastasis in breast cancer: A randomised study. Eur. J. Cancer 40(18), 2726-2733 (2004).

30. Mrugala MM, Kim B, Sharma A et al. Phase II study of systemic high-dose methotrexate and intrathecal liposomal cytarabine for treatment of leptomeningeal carcinomatosis from breast cancer. Clin. Breast Cancer 19(5), 311-316 (2019).

31. Niwińska A. Brain metastases as site of first and isolated recurrence of breast cancer: the role of systemic therapy after local treatment. Clin. Exp. Metastasis 33(7), 677-685 (2016).

32. Le Rhun E, Wallet J, Mailliez A et al. Intrathecal liposomal cytarabine plus systemic therapy versus systemic chemotherapy alone for newly diagnosed leptomeningeal metastasis from breast cancer. Neuro Oncol. 22(4), 524-538 (2020).

- DEPOSEIN trial, the first phase III in leptomeningeal disease in breast cancer that shows improvement in overall survival for patients treated with intrathecal treatment and systemic anticancer treatment.

33. Weigel R, Senn P, Weis J, Krauss JK. Severe complications after intrathecal methotrexate (MTX) for treatment of primary central nervous system lymphoma (PCNSL). Clin. Neurol. Neurosurg. 106(2), 82-87 (2004).

34. Nayar G, Ejikeme T, Chongsathidkiet $P$ et al. Leptomeningeal disease: current diagnostic and therapeutic strategies. Oncotarget 8(42), 73312-73328 (2017).

35. Byrnes DM, Vargas F, Dermarkarian C et al. Complications of intrathecal chemotherapy in adults: single-institution experience in 109 consecutive patients. J. Oncol. 2019, 4047617 (2019).

36. Cardoso F, Senkus E, Costa A et al. 4th ESO-ESMO international consensus guidelines for advanced breast cancer (ABC 4). Ann. Oncol. 29(8), 1634-1657 (2018).

37. Ripamonti C, Trippa F, Barone G, Maranzano E. Prevention and treatment of bone metastases in breast cancer. J. Clin. Med. 2(3), 151-175 (2013).

38. Robertson JFR, Bondarenko IM, Trishkina E et al. Fulvestrant $500 \mathrm{mg}$ versus anastrozole $1 \mathrm{mg}$ for hormone receptor-positive advanced breast cancer (FALCON): an international, randomised, double-blind, phase 3 trial. Lancet 388(10063), 2997-3005 (2016).

39. Coleman RE, Roodman GD, Smith MR et al. Clinical features of metastatic bone disease and risk of skeletal morbidity. Clin. Cancer Res. 12(20 Pt 2), 6243-6250 (2006). 
40. Figura NB, Rizk VT, Mohammadi H et al. Clinical outcomes of breast leptomeningeal disease treated with intrathecal trastuzumab, intrathecal chemotherapy, or whole brain radiation therapy. Breast Cancer Res. Treat. 175(3), 781-788 (2019).

41. Kumthekar P, Tang SC, Brenner AJ et al. ANG1005, a brain-penetrating peptide-drug conjugate, shows activity in patients with breast cancer with leptomeningeal carcinomatosis and recurrent brain metastases. Clin. Cancer Res. 26(12), 2789-2799 (2020).

42. Peereboom DM, Murphy C, Ahluwalia MS et al. Phase II trial of patupilone in patients with brain metastases from breast cancer. Neuro Oncol. 16(4), 579-583 (2014).

43. Wu PF, Lin $\mathrm{CH}, \mathrm{Kuo} \mathrm{CH}$ et al. A pilot study of bevacizumab combined with etoposide and cisplatin in breast cancer patients with leptomeningeal carcinomatosis. BMC Cancer 15, 299 (2015).

44. Pan Z, Yang G, He H et al. Concurrent radiotherapy and intrathecal methotrexate for treating leptomeningeal metastasis from solid tumors with adverse prognostic factors: A prospective and single-arm study. Int. J. Cancer. 139(8), 1864-1872 (2016).

45. Bergen ES, Berghoff AS, Medjedovic $\mathrm{M}$ et al. Continued endocrine therapy is associated with improved survival in patients with breast cancer brain metastases. Clin. Cancer Res. 25(9), 2737-2744 (2019).

-. A retrospective work that discusses and shows the role of endocrine therapy in CNS metastasis.

46. Almajed MM, Esfahani K, Pelmus M, Panasci L. Complete response and long-term survival of leptomeningeal carcinomatosis from breast cancer with maintenance endocrine therapy. BMJ Case Rep. 2016, bcr2016215525 (2016).

47. Zoghi B, Elledge R. Endocrine therapy for leptomeningeal metastases from ER-Positive breast cancer: Case report and a review of the literature. Breast J. 22(2), 218-223 (2016).

48. Lien EA, Wester K, Lonning PE, Solheim E, Ueland PM. Distribution of tamoxifen and metabolites into brain tissue and brain metastases in breast cancer patients. Br. J. Cancer. 63(4), 641-645 (1991).

49. Patel MM, Patel BM. Crossing the blood-brain barrier: recent advances in drug delivery to the brain. CNS Drugs 31(2), 109-133 (2017).

50. Miyajima M, Kusuhara H, Takahashi K et al. Investigation of the effect of active efflux at the blood-brain barrier on the distribution of nonsteroidal aromatase inhibitors in the central nervous system. J. Pharm. Sci. 102(9), 3309-3319 (2013).

51. Dave N, Gudelsky GA, Desai PB. The pharmacokinetics of letrozole in brain and brain tumor in rats with orthotopically implanted C6 glioma, assessed using intracerebral microdialysis. Cancer Chemother. Pharmacol. 72(2), 349-357 (2013).

52. Richardson WS, Wilson MC, Nishikawa J, Hayward RS. The well-built clinical question: a key to evidence-based decisions. ACP J. Club 123(3), A12-13 (1995).

53. Stewart LA, Clarke M, Rovers M et al. Preferred reporting items for a systematic review and meta-analysis of individual participant data: the PRISMA-IPD statement. JAMA 313(16), 1657-1665 (2015).

54. R Core Team. R: A language and environment for statistical computing. (2013) https://cran.r-project.org/doc/manuals/fullref man.pdf

55. Brastianos PK, Lee EQ, Cohen JV et al. Single-arm, open-label phase 2 trial of pembrolizumab in patients with leptomeningeal carcinomatosis. Nat. Med. 26(8), 1280-1284 (2020).

56. Kingston B, Kayhanian H, Brooks C et al. Treatment and prognosis of leptomeningeal disease secondary to metastatic breast cancer: A single-centre experience. Breast 36, 54-59 (2017).

57. Lee YC, Hsieh CC, Chuang JP, Li CY. The necessity of intrathecal chemotherapy for the treatment of breast cancer patients with leptomeningeal metastasis: A systematic review and pooled analysis. Curr. Prob. Cancer 41(5), 355-370 (2017).

58. Boogerd W. Response of leptomeningeal metastases from breast cancer to hormonal therapy. Neurology 56(3), 425-426 (2001).

59. Ozdogan M, Samur M, Bozcuk HS et al. Durable remission of leptomeningeal metastasis of breast cancer with letrozole: A case report and implications of biomarkers on treatment selection. Jpn. J. Clin. Oncol. 33(5), 229-231 (2003).

60. Peroukides S, Onyenadum A, Starakis I et al. Prolonged survival of neoplastic meningitis from breast cancer with letrozole and intrathecal methotrexate: A case report. J. Neurooncol. 101(3), 509-511 (2011).

61. Singh SK, Bansal VK, Wiley EL, Valyi-Nagy T, Villano JL. Long-term survival in an untreated patient with leptomeningeal carcinomatosis from breast primary. Clin. Neurol. Neurosurg. 115(3), 362-364 (2013).

62. Madgula IM, Hemmerdinger CM, Clark P. Metastatic breast cancer presenting as sequential cranial nerve palsy: A case report. J. Med. Case Rep. 8, 430 (2014).

63. Takanashi T, Hikino H, Makino Y, Murata Y. Durable clinical benefit of letrozole in leptomeningeal metastasis of breast cancer. Int. Cancer Conf. J. 8(4), 146-148 (2019).

64. Kapke JT, Schneidewend RJ, Jawa ZA et al. High-dose intravenous methotrexate in the management of breast cancer with leptomeningeal disease: case series and review of the literature. Hematol. Oncol. Stem Cell Ther. 12(4), 189-193 (2019).

65. Tolaney SM, Sahebjam S, Le Rhun E et al. A phase 2 study of abemaciclib in patients with brain metastases secondary to hormone receptor positive breast cancer. Clin. Cancer Res. doi:10.1158/1078-0432.CCR-20-1764 (2020) (Epub ahead of print).

66. Shah N, Mohammad AS, Saralkar P et al. Investigational chemotherapy and novel pharmacokinetic mechanisms for the treatment of breast cancer brain metastases. Pharmacol. Res. 132, 47-68 (2018).

67. Rick JW, Shahin M, Chandra A et al. Systemic therapy for brain metastases. Crit. Rev. Oncol. Hematol. 142, 44-50 (2019). 
68. Morikawa A, Jordan L, Rozner R et al. Characteristics and outcomes of patients with breast cancer with leptomeningeal metastasis. Clin. Breast Cancer 17(1), 23-28 (2017).

69. Sperduto PW, Kased N, Roberge D et al. The effect of tumor subtype on the time from primary diagnosis to development of brain metastases and survival in patients with breast cancer. J. Neurooncol. 112(3), 467-472 (2013).

70. Hulsbergen AFC, Lamba N, Claes A et al. Prognostic value of brain metastasis-free interval in patients with breast cancer brain metastases. World Neurosurg. 128, e157-e164 (2019).

71. Costa R, Carneiro BA, Wainwright DA et al. Developmental therapeutics for patients with breast cancer and central nervous system metastasis: Current landscape and future perspectives. Ann. Oncol. 28(1), 44-56 (2017).

72. Hulsbergen AFC, Claes A, Kavouridis VK et al. Subtype switching in breast cancer brain metastases: a multicenter analysis. Neuro Oncol. 22(8), 1173-1181 (2020).

- A multicenter study that describes incidence, predictors and survival outcomes of discordant receptors and associated subtype switching in brain metastasis of breast cancer.

73. Sperduto PW, Mesko S, Li J et al. Estrogen/progesterone receptor and HER2 discordance between primary tumor and brain metastases in breast cancer and its effect on treatment and survival. Neuro Oncol. 29(9), 2341-2386 (2020).

74. Navarro Martín LM, Ocaña Fernández A, Rodríguez Sánchez CA, Ruiz Martín I, Cruz Hernández JJ. Durable clinical benefit with exemestane in leptomeningeal metastasis of breast cancer. Clin. Transl. Oncol. 7(8), 358-360 (2005).

75. Raub TJ, Wishart GN, Kulanthaivel P et al. Brain exposure of two selective dual CDK4 and CDK6 inhibitors and the antitumor activity of CDK4 and CDK6 inhibition in combination with temozolomide in an intracranial glioblastoma xenograft. Drug Metab. Dispos. 43(9), 1360-1371 (2015).

76. Batalini F, Moulder SL, Winer EP, Rugo HS, Lin NU, Wulf GM. Response of brain metastases from PIK3CA-mutant breast cancer to alpelisib. JCO Precis. Oncol. 4, DOI: 10.1200/PO.19.00403 (2020).

77. Shariati M, Meric-Bernstam F. Targeting AKT for cancer therapy. Expert Opin. Investig. Drugs 28(11), 977-988 (2019).

78. Jones RH, Casbard A, Carucci $\mathrm{M}$ et al. Fulvestrant plus capivasertib versus placebo after relapse or progression on an aromatase inhibitor in metastatic, oestrogen receptor-positive breast cancer (FAKTION): a multicentre, randomised, controlled, phase 2 trial. Lancet Oncol. 21(3), 345-357 (2020).

79. Le Rhun E, Bertrand N, Dumont A et al. Identification of single nucleotide polymorphisms of the PI3K-AKT-mTOR pathway as a risk factor of central nervous system metastasis in metastatic breast cancer. Eur. J. Cancer 87, 189-198 (2017).

80. Fitzgerald DM, Muzikansky A, Pinto C et al. Association between PIK3CA mutation status and development of brain metastases in HR+/HER2- metastatic breast cancer. Ann. Oncol. 30(Suppl. 5), v110 (2019).

81. Rugo HS, Lerebours F, Ciruelos E et al. Alpelisib (ALP) + fulvestrant (FUL) in patients (pts) with PIK3CA-mutated (mut) hormone receptor-positive $(\mathrm{HR}+)$, human epidermal growth factor receptor 2-negative (HER2-) advanced breast cancer (ABC) previously treated with cyclin-dependent kinase 4/6 inh. J. Clin. Oncol. 38(Suppl. 15), 1006 (2020).

82. Shen Q, Sahin AA, Hess KR et al. Breast cancer with brain metastases: clinicopathologic features, survival, and paired biomarker analysis. Oncologist 20(5), 466-473 (2015).

83. van Bussel MTJ, Pluim D, Milojkovic Kerklaan B et al. Circulating epithelial tumor cell analysis in CSF in patients with leptomeningeal metastases. Neurology 94(5), e521-e528 (2020).

84. Chun BM, Page DB, McArthur HL. Combination immunotherapy strategies in breast cancer. Curr. Breast Cancer Rep. 11(4), 228-240 (2019).

-. This paper summarizes combination of immunotherapy strategies for the treatment of breast cancer, with a focus on metastatic disease.

85. Esteva FJ, Hubbard-Lucey VM, Tang J, Pusztai L. Immunotherapy and targeted therapy combinations in metastatic breast cancer. Lancet Oncol. 20(3), e175-e186 (2019). 